\title{
Cleaning of mildew on the optical lens with an economical and ordinary Diode laser
}

\author{
Xinji Gan \\ Beihua University, Jilin, 132011,China \\ Email:ganxinji@sina.com
}

Keywords: laser cleaning; midew; optical lens; Diode laser

\begin{abstract}
Laser cleaning of the mildew on the optics lens with economical and ordinary Diode laser has been studied in this paper. Mucor and Penicillium are the simples tested in the experiments. The mechanism of laser cleaning them is thermal effect through the interacting of photon and mildew. It is demonstrated that Mucor which contaminates lens is more easily cleaned off than Penicillium. With appropriate laser energy density and dosing time, it is possible to removal the mildew form the lenses without any damage . The paper recommends an economical, effective; environmentally-friendly cleaning method which is easy implemented in technology, which is worthy to be applied in the field of cleaning optical instruments.
\end{abstract}

\section{Introduction}

In order to improve optical transmittance of lenses, the makers of lenses usually deposit one or more layers of optical coating which is made up of inorganic or organic materials. [1]But they are difficult to clean in many industrial applied fields[2]. Due to humid and warm environment a variety of mildews would breed on these optical coatings. Mildew is the popular name of fungus that can be able to form a branched, lush mycelium. They are white, brown, gray, or a bright color (white hairy colony is Mucor, green as Penicillium, yellow as aspergillus). They reproduce by spores, which can drift in the air when ripping. So the parts in the optical system, which outer air can penetrate into, are possible to be contaminated by those fungi. The majority of fungus kinds are adaptable to the surroundings of temperature of $20^{\circ} \mathrm{C}, 85 \% \mathrm{RH}$ and the base that can provide a large amount of nutrients to them. Some optical coatings just contribute to offer these fungi abundant nutrition by their absorbing organics.

For the sake of cleaning up the mildew, the usual method is to disassemble the lens or optical system, then to wipe them away with bush and some kinds of dissolvent manually. This process would not only spend a lot of time, but also bring the optical lens accidental scuffing, which would be an expensive loss. The paper provides an effective, economic method to clean off the mildew of optical lens by laser irradiation, which can permit not to disassemble the optical system.

\section{Principle of laser cleaning up mildew}

The principle of cleaning up mildew by laser is neither dry cleaning nor wet cleaning in the conventional meaning[3][4]. The force that makes the mildew adhere to the surface of lens mainly includes Van der Waals' force or some chemical bond like as hydrogen bond, what kind of force it is in fact depends on the material coated on the lens surface. The principle of cleaning up mildew by laser is photodecomposition effect and thermal effect which would occur when the laser photons interact with biomacromolecules of the mildew each other[5]. Photodecomposition refers to a chemical reaction in which a chemical compound is broken down by photons. When the bonding energy of mildew molecule and lens material is less than the energy of laser photon, the bond is destroyed or weakened. This can lead to separate mildew and achieve the purpose of cleaning. This process would not injure the lens base owing to less heat produced on the surface. The energy level $\mathrm{E}$ of laser depends on the laser wavelength $\lambda, \mathrm{E}=\mathrm{hc} / \lambda$. The body of mildew is composed of microbial macromolecule, whose cell consists of fat layers and protein layers. The outer layer is 
protein and the inner layer is fat. Under laser irradiation, the mildew absorbs the laser energy that makes the temperature rise to the melting point, which causes the mildew burn, volatilize. Besides that, the plenty of water included in mildew would also evaporate and vaporized when absorbing energy from laser photon. Especially the volume of water steam would expand rapidly when vaporization occurs, which leads to micro explosion in the inner of mildew and decomposition of them due to the increased pressure in the inner of mildew.

\section{Cleaning up Mucor}

Mucor is the most popular kind of mildew that contaminates optical system in humid and warm environment. In order to clean off the Mucor effectively, the selected wavelength of laser should help to obtain high absorption rate, low reflectivity and low transmittance of laser energy for the Mucor. Out of consideration to reducing cost, a kind of Diode laser which wavelength is $405 \mathrm{~nm}$ is employed in this research. Its power is only $500 \mathrm{~mW}$. The laser operates inTEM00-mode, as shown in Figure 1. The beam diameter after focusing can reduce to $0.5 \mathrm{~mm}$, which can increase the power intensity to $25 \mathrm{~W} / \mathrm{cm}^{2}$.

A mechanical shutter is adopted to decide the dosing time for cleaning with a control method using a single chip microcomputer, as shown in Figure 2. It is very convenient to manipulate the shutter because only $12 \mathrm{~V}$ DC working voltage and a TTL level are required to control the dosing time of laser. In addition, the switching delay of the shutter is low to $10 \mathrm{~ms}$, which is advantageous to adjust the dosing time according to where is needed to be cleaned off.

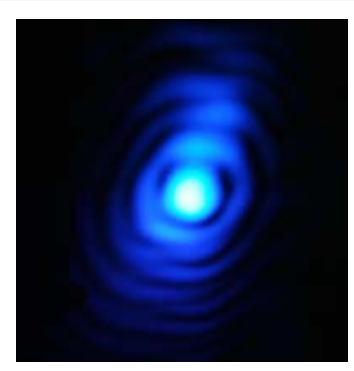

Figure 1. TEM 00 -mode of $405 \mathrm{~nm}$ Diode laser
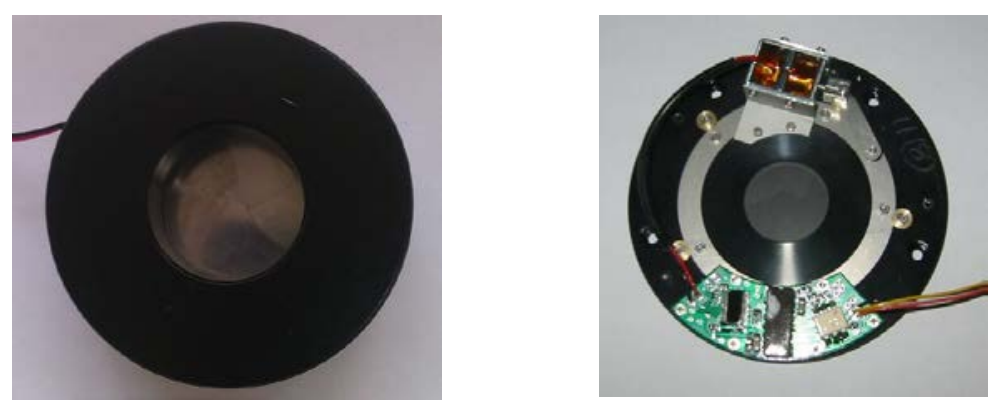

Figure 2. Mechanical shutter to control dosing time

The first experiment in the paper is to clean off Mucor that reproduce on the surface of an Optical attenuation lens. The Mucor was artificially propagated and has been dried before experimented, as shown in figure 3. The Mucor is covered in the whole surface of the lens. So it is difficult to clean when using a conditional method of scrubbing. In this cleaning experiment, five laser irradiation fields that arrange in a straight line on the lens surface with the equal space are tested in different dosing time from 1 to 5 seconds, as shown in Figure 4. The most left field is 
exposure with one second by focused laser beam. It can be seen the Mucor in the central zone is burned by laser, where almost all of Mucor has been cleaned off. The average diameter of cleaned field is $1.2 \mathrm{~mm}$. With the dosing time increasing, the diameter cleaned off become bigger. The figure 5 gives a graphical representation about the relation between the diameters of cleaned off field and the dosing time of laser. As is shown clearly from figure 5, although the areas of cleaned fields ascend with the dosing time, the rising rate of cleaned off areas start to fall when the dosing time is bigger than three seconds. The reason is that the energy density in outer region in the beam spot is smaller than the part of the central region. At the beginning stage of irradiation the heated temperature of Mucor is not enough high to make it burn. With the dosing time increasing, the Mucor of the outer region start to be cleaned off. But the whole area cleaned off would not exceed that of the beam spot. Figure 5 show the changes of cleaned diameter by laser with the dosing time.

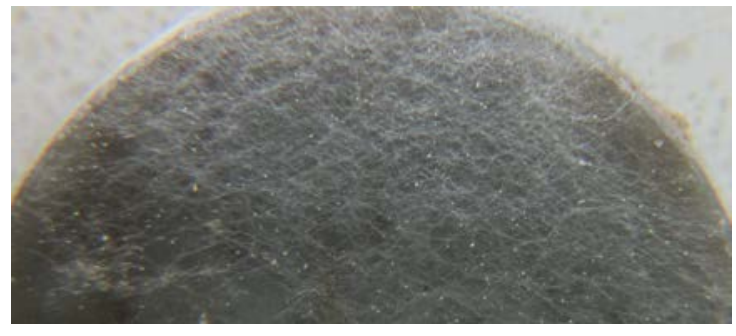

Figure 3. Lens covered by Mucor

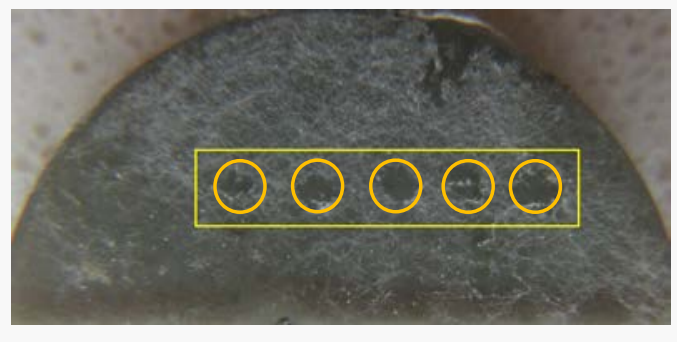

Figure 4. Five fields irradiated with different dosing time

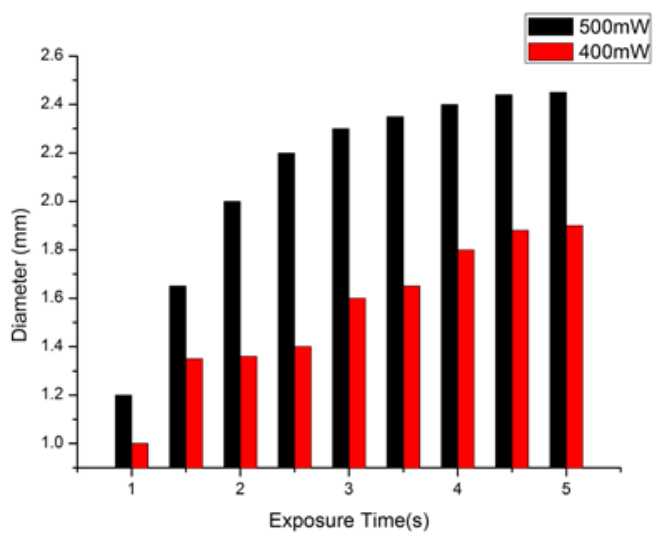

Figure 5. Relation between diameter of cleaned off field and dosing time

From the figure 5 it can be visible

As is shown clearly from figure 5 , the diameters of the cleaned off spots increase with the dosing time of laser.

\section{Cleaning up Penicillium}

Usually Penicillium would not exist on the surface of the lens as a part of precise instruments which is strictly protected in good conditions of lab. However, there still are their traces appearing in some optics lens that works in outer fields, for example, theodolites. In these cases, Penicillium more likely

grows on the outside of the optical lens. It is

noteworthy that most of such lenses belong to the kinds that are coated a layer of optical coating which contributes to increase the transmissivity or other optical capabilities of lenses. In this experiment for cleaning up Penicillium, a simple of lens whose one-sided surface contaminated by the fungi is used. The fungi cover the one surface of lens which is not coated. The other side has the coating film.

The laser employed in the experiment still is $405 \mathrm{~nm}$ Diode laser. The original Penicillium sample 
can be seen in figure6(a), which is circled in the picture. After the laser irradiating, the most of Penicillium obviously is burned and disappears, as shown in Figure 6(b). But there exists some residues left on the irradiated position. The cleaned rate relative to the dosing time is measured and the result is illustrated in figure 7.

It can be seen clearly that the clearance rate rise up with the dosing time increasing. The thickness of fungi also influences the result. The thicker the fungi are, the lower the clearance rates are. Three different cases of thickness are tested in the experiment.

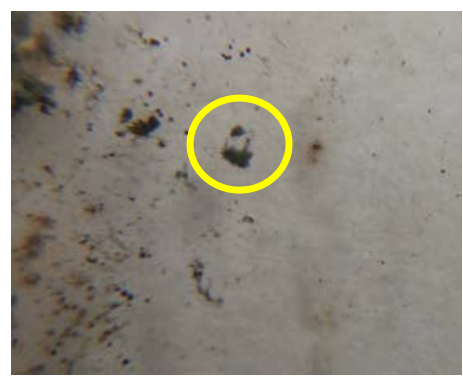

(a)

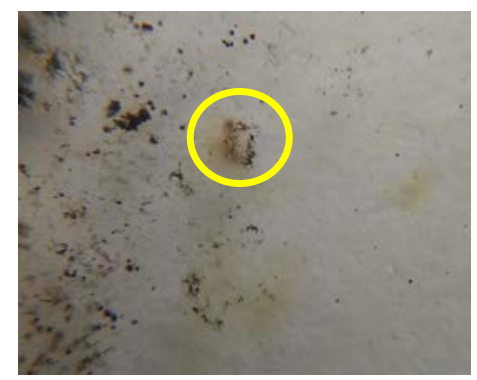

(b)

Figure 6. Original sample and cleaned sample of Penicillium

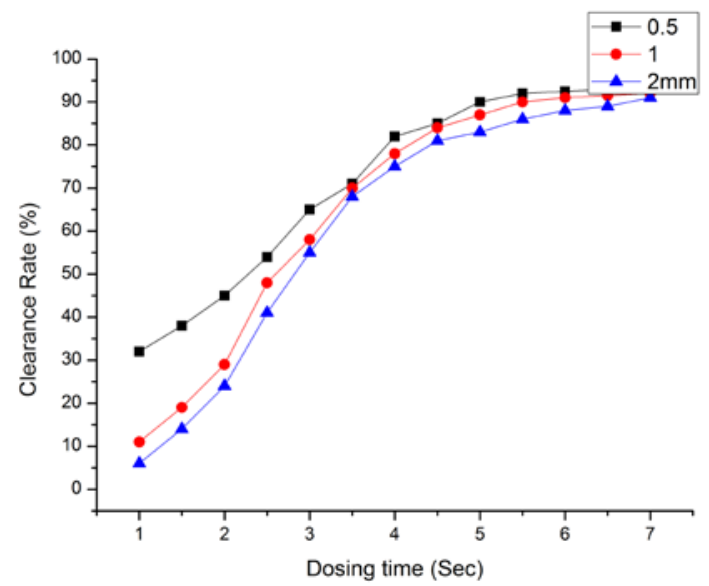

Figure 7. Relative curves of clearance rate of Penicillium and dosing time, sample thickness

Due to being thicker than Mucor in most cases, the process of cleaning take more time to burn the fungi, especially when it is needed to clean off thoroughly. It would often cause to a disastrous result. As shown in figure 8, the burnt color emerges on the back side of the irradiated spot, which means a permanent damage to the coating layer of lens. The heat effect destroys the brittle optical film completely, which can be seen more clearly after cleaned and washed with water ,as shown in figure 9 


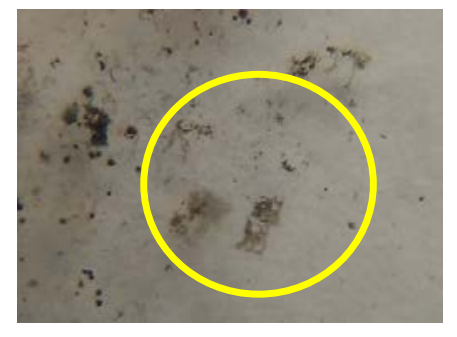

Figure 8. Position of coating layer nder long-time exposure

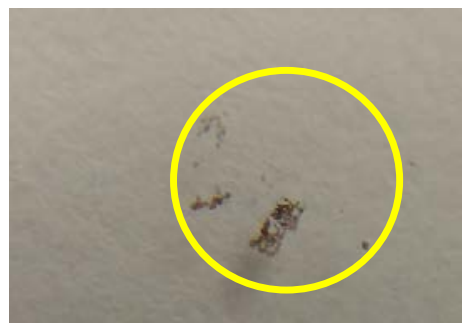

Figure 9. Damaged Coating layer

From the view of dosing time, Penicillium wastes more time to be burnt and cleaned off than Mucor. The reason is that the fat content included in Mucor is higher than Penicillium, which permits it to burn thoroughly with less time in one hand. In the other hand, the thickness of Mucor usually is thinner than that of Penicillium. So the time taken in burning them and cleaning them is shorter. Inversely, the Penicillium grows upward and has longer hypha. This makes them thicker. It has to take more times of exposure under laser beam to achieve the goal of cleaning up them. Therefore Mucor is more easily to be cleaned than Penicillium. It is more important that the permanent damage to optical coating materials on lens would be avoided due to shorter dosing time of laser.

\section{Conclusion}

The paper employees an economical and ordinary Diode laser to clean lenses contaminated by Mucor and Penicillium. There is different dosing time to clean off them with different kinds of fungi. Usually Mucor is more easily cleaned off than Penicillium. The optical coating layer is harder to be damaged permanently by intense laser at the same time. Considered to use such an economical laser and such an environmentally-friendly cleaning method which also is easy implemented in technology, the technique is worthy to be applied in the field of cleaning optical instruments.

\section{Acknowledgement}

In this paper, the research was sponsored by the Nature Science Foundation of Jilin Province (Project No. 201105040).

\section{References}

[1] W.D. Song a Y.F. Lu a Chen, etc. Laser-induced removal of organic contaminants from metal substrates [C]. SPIEVol. 3550 27-34.

[2] Pleasants S, Kane D M. Laser cleaning of aluminum particles on glass and silica substrates: Experiment and quasistaticmodel[J]. ApplPhys, 2003,93(11): 8862 8866.

[3] B.S. Luk'yanchuk, Y. W. Zheng, Y. F. Lu. A new mechanism of laser dry cleaning [C].Proceedings of SPIE Vol. 4423 (2001).115-126.

[4] Sergey Kudryashov, Shishir Shukla, Kevin Lyon. Advanced dry and steam laser cleaning of opaque and transparent critical substrates[C]. Proc. of SPIE Vol. 626162610 D1-D12.

[5] Mihai Oane, Ileana Apostol, Adrian Timcu. Temperature field modeling in laser heated metals for laser cleaning of surfaces.[C] Proceedings of SPIE Vol. 5227 (2003), 323-328. 